\title{
Ultra-low threshold gallium nitride photonic crystal nanobeam laser
}

\section{Citation}

Niu, Nan, Alexander Woolf, Danqing Wang, Tongtong Zhu, Qimin Quan, Rachel A. Oliver, and Evelyn L. Hu. 2015. "Ultra-Low Threshold Gallium Nitride Photonic Crystal Nanobeam Laser." Applied Physics Letters 106 (23) (June 8): 231104. Portico. doi:10.1063/1.4922211.

\section{Published Version}

doi:10.1063/1.4922211

\section{Permanent link}

http://nrs.harvard.edu/urn-3:HUL.InstRepos:27727028

\section{Terms of Use}

This article was downloaded from Harvard University's DASH repository, and is made available under the terms and conditions applicable to Other Posted Material, as set forth at http:// nrs.harvard.edu/urn-3:HUL.InstRepos:dash.current.terms-of-use\#LAA

\section{Share Your Story}

The Harvard community has made this article openly available.

Please share how this access benefits you. Submit a story.

Accessibility 


\title{
Ultra-low threshold gallium nitride photonic crystal nanobeam laser
}

\author{
Nan Niu, ${ }^{1, a)}$ Alexander Woolf, ${ }^{1}$ Danging Wang, ${ }^{1}$ Tongtong Zhu, ${ }^{2}$ Qimin Quan, ${ }^{3}$ \\ Rachel A. Oliver, ${ }^{2}$ and Evelyn L. Hu ${ }^{1}$ \\ ${ }^{1}$ School of Engineering and Applied Sciences, Harvard University, Cambridge, Massachusetts 02138, USA \\ ${ }^{2}$ Department of Materials Science and Metallurgy, University of Cambridge, 27 Charles Babbage Road, \\ Cambridge CB3 OFS, United Kingdom \\ ${ }^{3}$ Rowland Institute at Harvard University, Cambridge, Massachusetts 02142, USA
}

(Received 20 February 2015; accepted 26 May 2015; published online 10 June 2015)

\begin{abstract}
We report exceptionally low thresholds $\left(9.1 \mu \mathrm{J} / \mathrm{cm}^{2}\right)$ for room temperature lasing at $\sim 450 \mathrm{~nm}$ in optically pumped Gallium Nitride $(\mathrm{GaN})$ nanobeam cavity structures. The nanobeam cavity geometry provides high theoretical Q $(>100000)$ with small modal volume, leading to a high spontaneous emission factor, $\beta=0.94$. The active layer materials are Indium Gallium Nitride (InGaN) fragmented quantum wells (fQWs), a critical factor in achieving the low thresholds, which are an order-of-magnitude lower than obtainable with continuous QW active layers. We suggest that the extra confinement of photo-generated carriers for fQWs (compared to QWs) is responsible for the excellent performance. (C) 2015 AIP Publishing LLC. [http://dx.doi.org/10.1063/1.4922211]
\end{abstract}

Semiconductor nanocavities are excellent platforms for experimental studies of lasing dynamics and cavity QED. ${ }^{1-7}$ The large bandgap of the GaN-based materials offers great potential of highly efficient blue and UV emitting devices operating at room temperature. ${ }^{8-10}$ This work demonstrates a $\mathrm{GaN}$ photonic crystal (PC) nanobeam laser with a spontaneous emission factor, $\beta$, as high as 0.94 . The threshold of $50.1 \mu \mathrm{W}$ incident power, with an adjusted $9.1 \mu \mathrm{J} / \mathrm{cm}^{2}$ absorbed energy density marks a record low threshold for PC cavity lasers fabricated from $\mathrm{InGaN} / \mathrm{GaN}$ heterostructures alone. The active layer of these low-threshold structures consists of three fragmented quantum wells (fQWs): InGaN layers consisting of strips $50-100 \mathrm{~nm}$ wide, isolated by narrower troughs, filled with $\mathrm{GaN}$. The fragmented nature of the active layer has a dramatic influence on the lasing threshold: a continuous QW in the same nanobeam cavity produces lasers with an order of magnitude higher threshold. Because of the high surface area to volume ratio of the nanobeam cavities, the additional carrier confinement in the InGaN fQW active medium is essential in reducing non-radiative recombination with the sidewalls and surfaces of the nanobeam cavity. This results in dramatically improved lasing thresholds for the fQW nanobeam structure. As an active medium with greater carrier confinement than QWs, and higher carrier capture probability than quantum dots, the fQW thus provides an ideal means of probing the limits of light and matter interactions in a nanoscale cavity.

Nanobeam PC cavities offer high quality factors and small modal volumes, providing an ideal platform for realizing low threshold lasing. ${ }^{11,12}$ The particular cavity design utilized for these studies comprises a ridge waveguide perforated with gratings of circular holes designed using a deterministic high $Q$ method ${ }^{13,14}(\mathrm{Q}=$ quality factor). The cavity has a total length of $5.2 \mu \mathrm{m}$ with a hole periodicity of $130 \mathrm{~nm}$. The width and thickness of the beam is $125 \mathrm{~nm}$ and

\footnotetext{
${ }^{\text {a) }}$ Author to whom correspondence should be addressed. Electronic mail: nanniu@fas.harvard.edu
}

$200 \mathrm{~nm}$, respectively. Finite Difference Time Domain (FDTD) simulations of the intensity profile of the mode (Fig. 1(a)) indicate its confinement within the small volumes of the semiconductor material, between the inner etched holes. The simulations also indicate a resonance is at $419.48 \mathrm{~nm}$, with simulated Q factor of 101000 and modal volume $(\mathrm{V})$ of $1.7(\lambda / \mathrm{n})^{3}$, where $\mathrm{n}$ is 2.5 , the refractive index of $\mathrm{GaN}$ and $\lambda$ is the wavelength of operation of the device. The simulated value of $\mathrm{Q}$ will almost always be higher than the values obtained for fabricated structures, since the calculations do not take into account "real losses" due to scattering, absorption in the material or imperfections in the fabrication process. Nonetheless, it is important to begin with a design that offers a high theoretical value of $\mathrm{Q} / \mathrm{V}$.

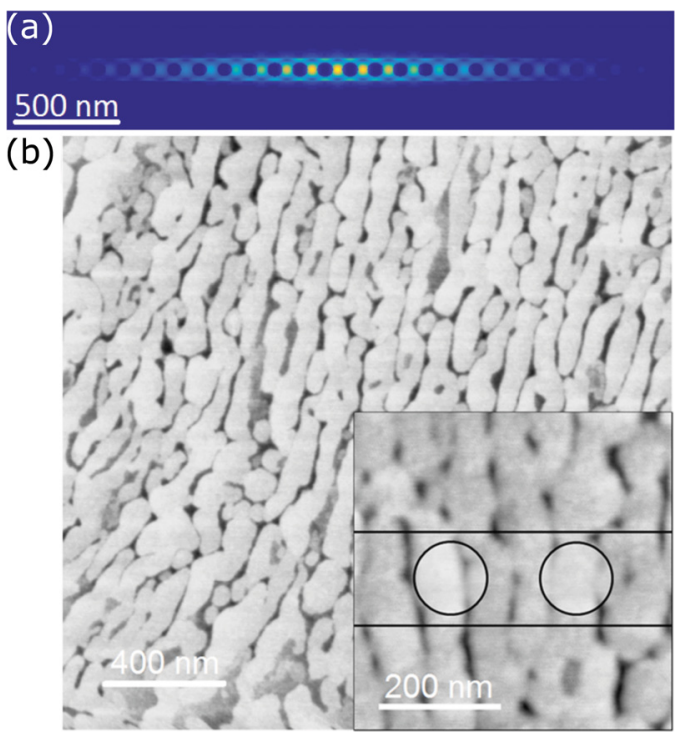

FIG. 1. (a) FDTD simulation of the intensity profile of mode at $419.48 \mathrm{~nm}$. The modal volume $(\mathrm{V})$ is $1.7(\lambda / \mathrm{n})^{3}$ and $\mathrm{Q}$ is approximately 101000 . The etched holes are circular. (b) AFM scan of the annealed InGaN epilayer showing the fQWs before capping. The inset image presents a possible overlay of the nanobeam and the fQW structures at the same size scale, showing the interplay of the two. 
To form the cavity structure and the active layer material, metalorganic vapor phase epitaxy (MOVPE) was used, beginning with an n-doped $c$-plane $\mathrm{GaN} / \mathrm{Al}_{2} \mathrm{O}_{3}$ pseudo-substrate (typical dislocation density is ca. $3.5 \times 10^{8} \mathrm{~cm}^{-2}$ ). ${ }^{15,16} \mathrm{~A}$ $200 \mathrm{~nm}$ thick $\operatorname{In}_{\mathrm{x}} \mathrm{Ga}_{1-\mathrm{x}} \mathrm{N} / \mathrm{In}_{\mathrm{y}} \mathrm{Ga}_{1-\mathrm{y}} \mathrm{N}$ sacrificial superlattice (SSL, $\mathrm{x}=6.5 \%, \mathrm{y}=5 \%$ ) was grown and capped by a thin $(\sim 10 \mathrm{~nm}) \mathrm{GaN}$ layer, followed by a $20 \mathrm{~nm} \mathrm{Al}_{0.2} \mathrm{Ga}_{0.8} \mathrm{~N}$ etch stop and a $180 \mathrm{~nm}$ thick GaN membrane containing the InGaN/GaN active layers composed of three fragmented InGaN QWs. Each fQW was formed by growing a $2.5 \mathrm{~nm}$ thick InGaN epilayer at $710^{\circ} \mathrm{C}$ and annealing at the growth temperature for $240 \mathrm{~s}$ in an atmosphere of $\mathrm{NH}_{3}$ and $\mathrm{N}_{2}$ prior to capping with $7.5 \mathrm{~nm}$ of GaN. Following annealing, the InGaN epilayer exhibits a network of interlinking InGaN strips aligned roughly along the [11-20] direction ${ }^{13}$ as shown in Fig. 1(b), an AFM scan of the fQW epilayer prior to the growth of the GaN cap. The average width of the InGaN strips is approximately $70 \mathrm{~nm}$. After the growth of the GaN capping layer, a composition gradient in the InGaN strip is expected to form, making the center of the strip more indium rich than the edges. ${ }^{17}$ This creates a graded electronic potential which confines the carriers at the center of the strips. For comparison, analogous structures were grown that contained three layers of continuous InGaN QW material. Both the QWs and the $\mathrm{GaN}$ barriers were grown at a temperature of $740^{\circ} \mathrm{C}$, again under $\mathrm{N}_{2}$. The differences in the growth temperatures of the fQW and QW samples allowed similar peak emission wavelengths from the two structures, $\sim(450 \pm 5) \mathrm{nm}$. Both the fQW and QW samples exhibited negligible surface roughness. $\mathrm{X}$-ray diffraction (XRD) was used to characterize the indium content of continuous QWs to be approximately $18 \%$. Reliable XRD quantification of the indium composition of fQWs is difficult due to their non-uniformity. While the average indium content of the fQW will be lower than that of the QW, our previous microscopy studies suggest that at the center of the InGaN strips both the width and composition of the fQW should be similar to that of the QW structure. Photoluminescence measurements revealed the as-grown QW sample to be approximately twice as bright as the fQW sample, likely due to the larger amount of InGaN material present in the continuous QWs. The full width at half maximum (FWHM) of the fQW sample is $35 \mathrm{~nm}$, approximately $5 \mathrm{~nm}$ broader than that of the QW sample, most likely due to the inhomogeneous broadening originating from the uncontrollable size variations in the fQWs.

Fabrication of the photonic crystal nanobeams was carried out in a two-step dry etching transfer process followed by a photoelectrochemical (PEC) etch process to undercut the nanobeams and optically isolate them from the substrate. First, $5 \mathrm{~nm}$ of $\mathrm{SiO}_{2}$ is deposited on the as-grown $\mathrm{GaN} / \mathrm{InGaN}$ wafer prior to evaporation of $15 \mathrm{~nm}$ of $\mathrm{Ti}$ as conductive layer. Then negative resist (XR-1541) is spin-coated and E-beam lithography (Elionix F-125) is used to define the nanobeam and circular pad which served as masks for the subsequent inductively coupled plasma (ICP) etch in 25 sccm of $\mathrm{N}_{2}$ and $\mathrm{Cl}_{2}$ gas for an approximate depth of $300 \mathrm{~nm}$. Subsequently, FOx-16 resist is spin-coated and E-beam lithography is again used to define a large rectangular pad aligned to the dry-etched nanobeam and circular pad. This pattern was subsequently dry-etched to a depth of approximately $200 \mathrm{~nm}$, using the same conditions as described above. This allows access to the $\operatorname{In}_{x} \mathrm{Ga}_{1-\mathrm{x}} \mathrm{N} / \mathrm{In}_{\mathrm{y}} \mathrm{Ga}_{1-\mathrm{y}} \mathrm{N}$ superlattice, which is then selectively removed by the PEC etch in a solution of $0.004 \mathrm{M} \mathrm{HCl}$. This produces the final suspended photonic crystal structure shown in Figs. 2(a) and 2(b): the top-down and side-view scanning electron microscope (SEM) images of the photonic crystal nanobeam device. Details of the PEC process can be found elsewhere. ${ }^{18}$ The InGaN fQW active layer is located in the middle of the cavity membrane, sandwiched by the GaN barrier layers.

Optical characterization of the nanobeams was performed using a frequency-doubled pulsed titanium-sapphire laser focused onto the sample through a long working distance objective $(\times 40, \mathrm{NA}=0.5)$. The source produces pulses of $380 \mathrm{~nm}$ light ( $200 \mathrm{ps}$ pulse length and $76 \mathrm{MHz}$ repetition rate), an energy below the bandgap of GaN and above the bandgap of the InGaN fQWs. We assume that all incident excitation power is focused onto the device and that the laser spot is a uniform Gaussian shaped beam $290 \mathrm{~nm}$ in radius.

The Qs of the fabricated devices can be found by calculating $\lambda / \Delta \lambda$ of the mode peak; the $Q$ values range from 1300 to 1900 . The etched holes of the fabricated device were slightly smaller than the designed values, resulting in a mode positioned around $454 \mathrm{~nm}$. This actually better matched the gain medium, which displayed a peak at $\sim 460 \mathrm{~nm}$. Lasing behavior in the nanobeam devices is clearly demonstrated through the linewidth narrowing $(0.32 \mathrm{~nm}$ to $0.1 \mathrm{~nm}$ at the onset of lasing) and the dramatic increase of the PL emission intensity as a function of incident pump power. ${ }^{19}$ Three different spectra are shown in Fig. 3(a), taken below threshold, at threshold, and above threshold. The inset image of Fig. 3(a) compares the discernible narrowing in linewidth of the principal mode when excited below and above threshold. The broad background at low pump power is the signature emission of the active medium, coupled to the leaky modes

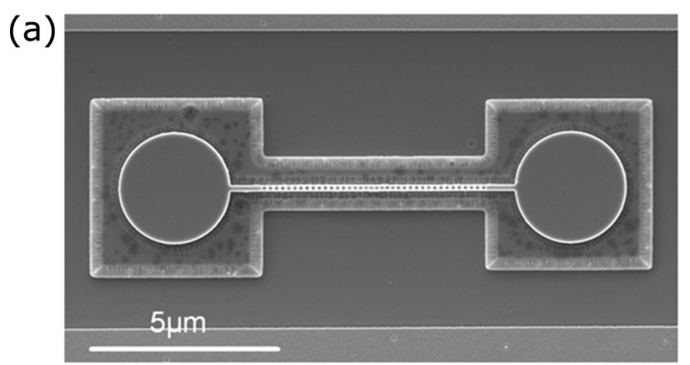

(b)

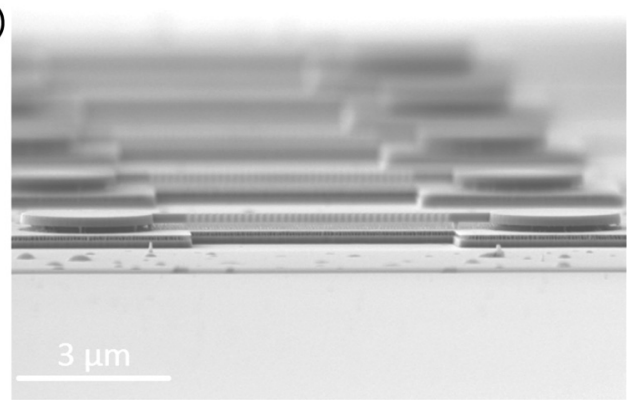

FIG. 2. (a) SEM top-view image of the photonic crystal nanobeam and (b) SEM side-view image of the photonic crystal nanobeam. 

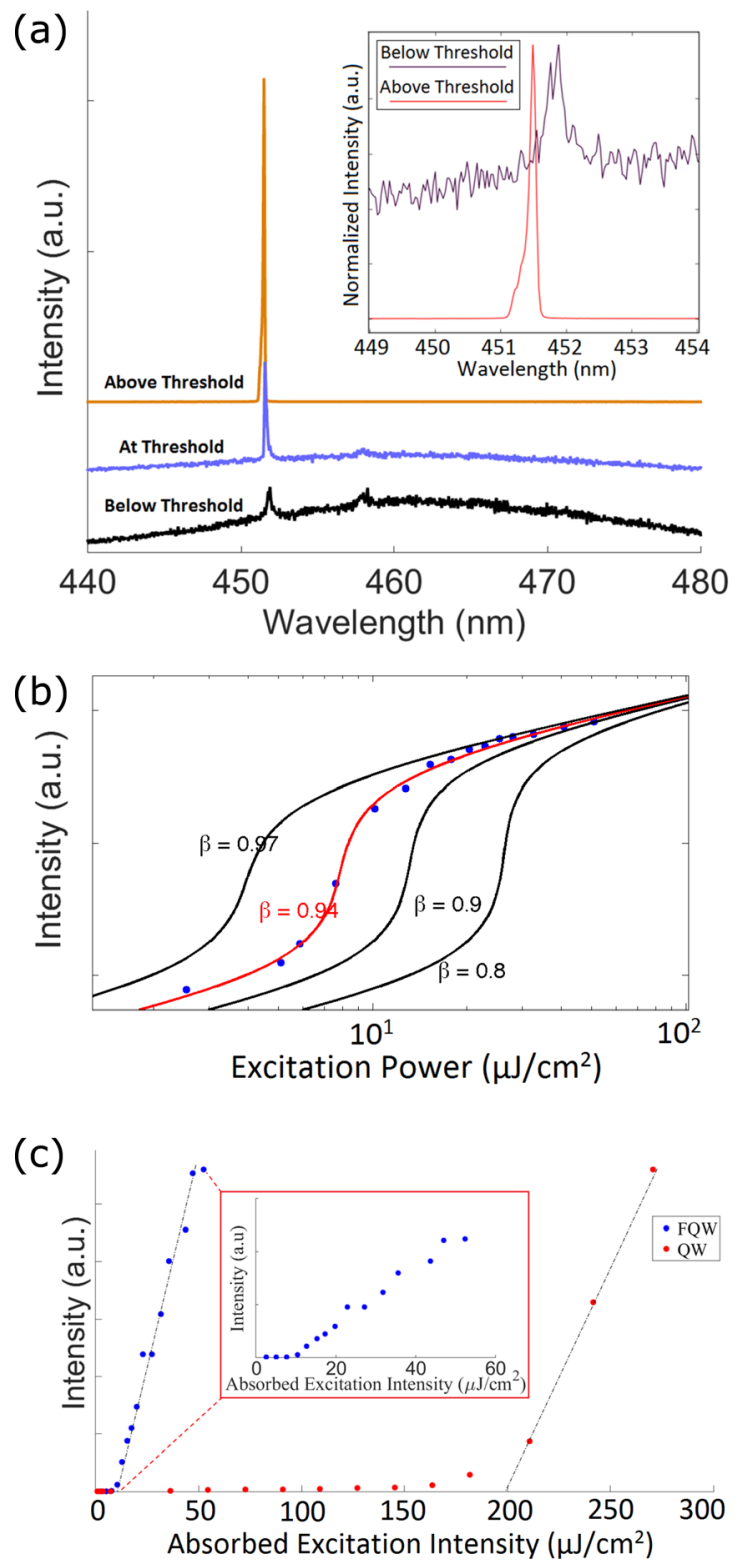

FIG. 3. (a) Spectra of the nanobeam at three pump regimes: below threshold, at threshold, and above threshold. The spectra taken with pump power above and at threshold are attenuated 1250 times and 5 times, respectively, with respect to the spectrum taken with below threshold pumping. The inset graph shows a discernible narrowing in the linewidth of the principal mode when excited below and above threshold. (b) Log-log plot of the emission intensity vs. pump power clearly indicating three regions of lasing operation. (c) Linear output intensity vs. pump power plots comparing the thresholds of the fQW and QW lasers. The inset shows a zoomed-in version of the plot for the fQW laser with $9.1 \mu \mathrm{J} / \mathrm{cm}^{2}$ threshold. The QW laser has an adjusted threshold of $198.6 \mu \mathrm{J} / \mathrm{cm}^{2}$.

of the cavity. At increased pump power, there is a slight blue-shift of the peak, which may be related to screening of the built-in electric field. Fig. 3(b) provides a log-log plot of the output intensity versus input power for the device with the lowest threshold, demonstrating all three regimes of operation: spontaneous emission, amplified spontaneous emission, and laser oscillation. ${ }^{20}$ Fits of these data to the laser rate equations suggest a spontaneous emission factor as high as $\beta=0.94$. The high beta results from the small modal volume, the high quality factor of the mode and the overlap of that single mode with the gain region. The result is an efficient channeling of the emitted light into essentially a single mode.

We estimate that the fraction of incident pump power absorbed by the device is approximately $0.9 \%$, assuming a value of absorption coefficient $5 \times 10^{6}\left(\mathrm{~m}^{-1}\right)^{21}$ with an average InGaN fQW thickness of approximately $2.5 \mathrm{~nm}$. Accounting for an exciting beam with diameter $(290 \mathrm{~nm})$ larger than the width of the nanobeam, and for some reflection of the incident light, we calculate adjusted lasing thresholds as low as $9.1 \mu \mathrm{J} / \mathrm{cm}^{2}$. The calculations assume that the incident power is absorbed in the InGaN layers which have a nominal total thickness of $7.5 \mathrm{~nm}$. Given the possibility of some absorption centers in the GaN layers, a conservative estimate of the absorption depth would be $10 \%$ of the thickness of the nanobeam or $20 \mathrm{~nm}$. This would increase the adjusted thresholds by roughly a factor of 2.6, indicating the range of error in our calculation. At pump powers far above the lasing threshold, the slope of the curve of laser intensity versus pump power levels off, as is shown in the inset of Fig. 3 (c), and the linewidth of the lasing mode is broadened to approximately 1.6 times the linewidth at the onset of lasing. The broadening may indicate heating effects and the generation of excess carriers.

Nanobeam structures identical to those previously described, but which incorporated three layers of continuous QW material of approximately the same composition showed a far poorer percentage of lasing devices. While all of the $11 \mathrm{fQW}$ nanobeam lasers probed demonstrated lasing, only 3 out of 10 of the continuous QW nanobeam devices, with minimum measured $\mathrm{Q}$ of $\sim 1000$, showed clear lasing behavior using the excitation power allowed by our optical set-up. The variations in the gain medium quality across the sample wafer give rise to the variation in thresholds observed for both fQW and QW lasers. For the much higher thresholds needed to achieve lasing in the QW structures, heating of the nanobeam structures and effects such as free carrier absorption may explain the lower percentage of successful lasing in the QW devices.

For 5 fQW nanobeams that were analyzed in detail, the lasing thresholds range from $9.1 \mu \mathrm{J} / \mathrm{cm}^{2}$ to $27.2 \mu \mathrm{J} / \mathrm{cm}^{2}$ with an average of $15.6 \mu \mathrm{J} / \mathrm{cm}^{2}$, and the lasing wavelengths are relatively consistent at around $454 \mathrm{~nm}$. Comparatively, the average adjusted threshold for the QW nanobeam lasers is $203.6 \mu \mathrm{J} / \mathrm{cm}^{2}$, more than an order of magnitude higher than the average threshold of the fQW nanobeam lasers. Fig. 3(c) shows the linear output intensity vs. pump power plots for the fQW and QW lasers, demonstrating clear lasing behavior with a dramatic difference in thresholds. These results are particularly interesting since our earlier comparison of lasing thresholds in microdisk cavities with $1.2 \mu \mathrm{m}$ diameter yielded the opposite outcome: the average lasing threshold for cavities with fQW active layers was approximately four times greater than for cavities with QW active layers. In addition, the range of threshold powers was about an order of magnitude larger for the fQW microcavity lasers. For both samples, no correlation between the lasing threshold and Q factor is observed, similar to the experimental observation on the microdisk lasers. ${ }^{19}$ 
We believe that the difference results from the changes in the relative loss mechanisms for carriers and photons as the cavity-active medium system is altered. The nanobeam cavities provide smaller mode volumes than do the microdisks, allowing a stronger mode-emitter coupling. The inset of Fig. 1(b) shows a portion of the nanobeam cavity dimensions overlaid on the as-grown annealed InGaN epilayer, illustrating the interplay of the scales of the two structures. The schematic shown in Fig. 4(a) suggests how electron-hole pairs created within a continuous QW active layer of the nanobeam cavity may diffuse to the edges of the etched holes and recombine non-radiatively with surface states. The region enclosed by the dashed line delineates the approximate boundaries of the center cavity mode. If the average distance of photo-created carrier to etched surface is less than a carrier diffusion length $\left(\mathrm{L}_{\mathrm{D}}\right)$, then we would expect substantial loss to non-radiative recombination. Although we have not measured the diffusion lengths and radiative lifetimes of these particular samples, we may find guidance from the literature in order to make an order-of-magnitude estimate of $L_{D}=[D t]^{1 / 2}$, where $D$ is the diffusion constant, and $t$ is the carrier lifetime for the InGaN active material. Danhof et al. ${ }^{22}$ carried out time-of-flight measurements of carrier diffusion in InGaN/GaN QWs. They deduced an ambipolar diffusion constant at room temperature of $1.2 \mathrm{~cm}^{2} / \mathrm{s}$ for InGaN QWs emitting at $470 \mathrm{~nm}$. Values of $\mathrm{t}$ will also vary, depending on the nature and quality of the active layer material, but we can use an order of magnitude estimate of $t \sim 10 \mathrm{~ns}^{23}$ The resulting estimate for $L_{D}$ is $\sim 1.1 \mu \mathrm{m}$, while the typical distance between holes in our nanobeam is $\sim 130 \mathrm{~nm}$. Thus, a substantial proportion of the photo-generated carriers will diffuse to the edges or surfaces of the nanobeam and undergo non-radiative recombination with surface states. The large reduction in carrier-generated photons leads to significantly increased lasing thresholds for nanobeams with continuous QW active layers. By contrast, the modulated potential barriers of the fQW active layers enhance the localization of the carriers and limit diffusion to the surfaces as illustrated by Fig. 4(b). We note that earlier work on as-grown fQW materials demonstrated higher PL efficiency than continuous QW material at low excitation powers. The reason underlying this behavior, impeding carrier diffusion to dislocations with subsequent non-radiative recombination, supports the role of the fQWs in these lowthreshold nanobeam lasers. ${ }^{24,25}$ (a)

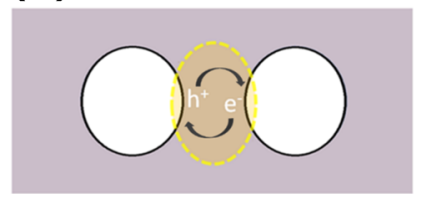

(b)

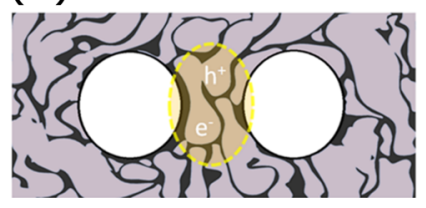

FIG. 4. (a) Schematic of a nanobeam cavity with continuous InGaN QW active medium. Photo-generated electron-hole pairs can diffuse to the edges of the etched holes and recombine non-radiatively with surface states as indicated by the arrows. (b) Schematic of a nanobeam cavity with InGaN fQW active medium. Photo-generated electron-hole pairs are localized within the boundaries of the isolated islands of the fQW. The regions enclosed by the dashed line in (a) and (b) represent the boundary of the center cavity mode.
In contrast, the best coupling between microdisk cavity and active layer for the multiple maxima distributed along the periphery of the microdisk is achieved for a gain medium that is as uniform as possible. ${ }^{26}$ Carriers generated through the entire interior of the disk may diffuse to the periphery, recombine radiatively and interact with the whispering gallery modes. The larger lateral dimensions of the microdisk cavity (with radius $>\mathrm{L}_{\mathrm{D}}$ ) allow the collection of photons from a larger fraction of the photo-generated carriers. ${ }^{27}$ The spatial variability of the fQW material disrupts that uniformity, leading to a larger variability and often larger value of the lasing threshold. Thus, the continuous QW active layers produce lower threshold lasing than fQW active layers for microdisk cavities. Future work will explore the further details of the different behavior of the fQW and QW active layers within microdisk and nanobeam cavities.

In conclusion, we have demonstrated an ultra-low threshold InGaN/GaN photonic crystal nanobeam laser. A clear transition from spontaneous emission to lasing is observed with clear linewidth narrowing. The ultimate device has an adjusted threshold of $9.1 \mu \mathrm{J} / \mathrm{cm}^{2}$. The ultra-small modal volume of the cavity and the reduction in the number of competing modes are useful in reducing the threshold. Moreover, we observed an order of magnitude reduction in lasing threshold on nanobeams fabricated from fQWs, which exhibit nanoscale non-uniformity, compared to continuous QWs because of an increased carrier confinement which is expected to reduce the impact of surface states. These observations underscore the advantages of this photonic crystal nanobeam design, matched to the 3-layer fQW gain material. Matching nanocavity geometry to the nanostructured gain medium provides $\mathrm{GaN} / \mathrm{InGaN}$ lasers with excellent performance. Because of their compact size and low thresholds, these devices are excellent candidates for efficient, on-chip optical sources in the blue portion of the spectrum.

The authors thank Dr. Tsung-li Liu and Dr. Jonathan Lee for useful discussions and Dr. Yinan Zhang for initial assistance with the FDTD simulations. This work was enabled by facilities available at the Center for Nanoscale Systems (CNS), a member of the National Nanotechnology Infrastructure Network (NNIN), which was supported by the National Science Foundation under NSF Award No. ECS0335765. This work was also supported in part by the NSF Materials World Network (Award No. 1008480), the Engineering and Physical Sciences Research Council (Award No. EP/H047816/1), and the Royal Academy of Engineering.

${ }^{1}$ M. S. Skolnick, T. A. Fisher, and D. M. Whittaker, Semicond. Sci. Technol. 13, 645 (1998).

${ }^{2}$ E. Peter, P. Senellart, D. Martrou, A. Lemaître, J. Hours, J. Gérard, and J. Bloch, Phys. Rev. Lett. 95, 067401 (2005).

${ }^{3}$ K. Hennessy, A. Badolato, M. Winger, D. Gerace, M. Atatüre, S. Gulde, S. Fält, E. L. Hu, and A. Imamoğlu, Nature 445, 896-899 (2007).

${ }^{4}$ N. Niu, T. L. Liu, I. Aharonovich, K. J. Russell, A. Woolf, T. C. Sadler, H. A. R. El-Ella, M. J. Kappers, R. A. Oliver, and E. L. Hu, Appl. Phys. Lett. 101, 161105 (2012).

${ }^{5}$ M. Pelton, C. Santori, J. Vuckovic, B. Zhang, G. S. Solomon, J. Plant, and Y. Yamamoto, Phys. Rev. Lett. 89, 233602 (2002). 
${ }^{6}$ P. Michler, A. Kiraz, C. Becher, W. V. Schoenfeld, P. M. Petroff, L. Zhang, E. L. Hu, and A. Imamoglu, Science. 290, 2282-2285 (2000).

${ }^{7}$ A. Kiraz, M. Atatüre, and A. Imamoğlu, Phys. Rev. A. 69, 032305 (2004).

${ }^{8}$ C. C. Chen, M. H. Shih, Y. C. Yang, and H. C. Kuo, Appl. Phys. Lett. 96, 151115 (2010).

${ }^{9}$ A. C. Tamboli, E. D. Haberer, R. Sharma, K. H. Lee, S. Nakamura, and E. L. Hu, Nat. Photonics 1, 61-64 (2006).

${ }^{10}$ S. Chang, N. B. Rex, R. K. Chang, G. Chong, and L. J. Guido, Appl. Phys. Lett. 75, 166 (1999).

${ }^{11}$ Y. Gong, B. Ellis, G. Shambat, T. Sarmiento, J. S. Harris, and J. Vučković, Opt. Express 18, 8781-8789 (2010).

${ }^{12}$ Y. Zhang, M. Khan, Y. Huang, J. H. Ryou, P. B. Deotare, R. Dupuis, and M. Lončar, Appl. Phys. Lett. 97, 051104 (2010).

${ }^{13}$ Q. Quan, P. B. Deotare, and M. Loncar, Appl. Phys. Lett. 96, 203102 (2010).

${ }^{14}$ Q. Quan and M. Loncar, Opt. Express 19, 18529-18542 (2011).

${ }^{15}$ R. A. Oliver, M. J. Kappers, J. Sumner, R. Datta, and C. J. Humphreys, J. Cryst. Growth 289, 506-514 (2006).

${ }^{16}$ R. Datta, M. J. Kappers, M. E. Vickers, J. S. Barnard, and C. J. Humphreys, Superlattices Microstruct. 36, 393-401 (2004).

${ }^{17}$ N. K. van der Laak, R. A. Oliver, M. J. Kappers, and C. J. Humphreys, J. Appl. Phys. 102, 013513 (2007).
${ }^{18}$ E. D. Haberer, R. Sharma, A. R. Stonas, S. Nakamura, S. P. DenBaars, and E. L. Hu, Appl. Phys. Lett. 85, 762 (2004).

${ }^{19}$ I. Aharonovich, A. Woolf, K. J. Russell, T. Zhu, N. Niu, M. J. Kappers, R. A. Oliver, and E. L. Hu, Appl. Phys. Lett. 103, 021112 (2013).

${ }^{20}$ A. E. Siegman, Lasers (University Science Books, Mill Valley, CA, 1986).

${ }^{21}$ M. Kuball, E. S. Jeon, Y. K. Song, A. V. Nurmikko, and P. Kozodoy, Appl. Phys. Lett. 70, 2580 (1997).

${ }^{22}$ J. Danhof, U. T. Schwarz, A. Kaneta, and Y. Kawakami, Phys. Rev. B 84, 035324 (2011).

${ }^{23}$ J. Danhof, H. M. Solowan, U. T. Schwarz, A. Kaneta, Y. Kawakami, D. Schiavon, T. Meyer, and M. Peter, Phys. Status Solidi B 249, 480-484 (2012).

${ }^{24}$ R. A. Oliver, F. C.-P. Massabuau, M. J. Kappers, W. A. Phillips, E. J. Thrush, C. C. Tartan, W. E. Blenkhorn, T. J. Badcock, P. Dawson, M. A. Hopkins, D. W. E. Allsopp, and C. J. Humphreys, Appl. Phys. Lett. 103, 141114 (2013).

${ }^{25}$ F. C.-P. Massabuau, C. C. Tartan, R. Traynier, W. E. Blenkhorn, M. J. Kappers, P. Dawson, C. J. Humphreys, and R. A. Oliver, J. Cryst. Growth 386, 88-93 (2014).

${ }^{26}$ A. Woolf, T. Puchtler, I. Aharonovich, T. Zhu, R. A. Oliver, and E. L. Hu, Proc. Natl. Acad. Sci. U.S.A. 111, 14042-14046 (2014).

${ }^{27}$ T. Baba, IEEE J. Sel. Top. Quantum Electron. 3, 808-830 (1997). 